\title{
The ICR142 NGS validation series: a resource for orthogonal assessment of NGS analysis [version 1; peer review: 2
}

\section{approved]}

\author{
Elise Ruark ${ }^{1}$, Anthony Renwick ${ }^{1}$, Matthew Clarke1, Katie Snape1, Emma Ramsay ${ }^{1}$, \\ Anna Elliott ${ }^{1}$, Sandra Hanks ${ }^{1}$, Ann Strydom ${ }^{1}$, Sheila Seal ${ }^{1}$, Nazneen Rahman ${ }^{1,2}$ \\ ${ }^{1}$ Division of Genetics \& Epidemiology, The Institute of Cancer Research, London, UK \\ ${ }^{2}$ Cancer Genetics Unit, Royal Marsden NHS Foundation Trust, London, UK
}

\section{V1 First published: 22 Mar 2016, 5:386 \\ https://doi.org/10.12688/f1000research.8219.1}

Latest published: 05 Sep 2018, 5:386

https://doi.org/10.12688/f1000research.8219.2

\section{Abstract}

To provide a useful community resource for orthogonal assessment of NGS analysis software, we present the ICR142 NGS validation series. The dataset includes high-quality exome sequence data from 142 samples together with Sanger sequence data at 730 sites; 409 sites with variants and 321 sites at which variants were called by an NGS analysis tool, but no variant is present in the corresponding Sanger sequence. The dataset includes 286 indel variants and 275 negative indel sites, and thus the ICR142 validation dataset is of particular utility in evaluating indel calling performance. The FASTQ files and Sanger sequence results can be accessed in the European Genomephenome Archive under the accession number EGAS00001001332.

Keywords

Variant calling , next-generation sequencing , NGS, exome , indel , validation

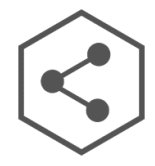

This article is included in the Data: Use and Reuse collection.

\section{Open Peer Review}

Approval Status

1

2

version 2

(revision)

05 Sep 2018

version 1

22 Mar 2016

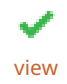

view

1. Richard Bagnall, The University of Sydney,

Sydney, Australia

2. Brad Chapman, Harvard Public School of

Health, Boston, USA

Oliver Hofmann, University of Glasgow, Glasgow, UK

Any reports and responses or comments on the article can be found at the end of the article. 
Corresponding author: Nazneen Rahman (drnazneenrahman100@gmail.com)

Competing interests: No competing interests were disclosed.

Grant information: We acknowledge NHS funding to the NIHR Biomedical Research Centre at The Royal Marsden and the ICR. This study was funded by the Institute of Cancer Research, London.

The funders had no role in study design, data collection and analysis, decision to publish, or preparation of the manuscript.

Copyright: @ 2016 Ruark E et al. This is an open access article distributed under the terms of the Creative Commons Attribution License, which permits unrestricted use, distribution, and reproduction in any medium, provided the original work is properly cited.

How to cite this article: Ruark E, Renwick A, Clarke M et al. The ICR142 NGS validation series: a resource for orthogonal assessment of NGS analysis [version 1; peer review: 2 approved] F1000Research 2016, 5:386 https://doi.org/10.12688/f1000research.8219.1

First published: 22 Mar 2016, 5:386 https://doi.org/10.12688/f1000research.8219.1 


\section{Introduction}

Next-generation sequencing (NGS) approaches have greatly enhanced our ability to detect genetic variation. Over the past decade NGS hardware, software, throughput, data quality and analytical tools have evolved dramatically. Thorough evaluation of each new laboratory and analytical development is challenging but necessary to fully understand how pipeline modification can impact results. To fully assess performance, NGS analysis tools should ideally be run on samples with pre-determined positive and negative sites assessed through orthogonal experimentation such as Sanger sequencing.

Over the past five years, we have generated extensive data on thousands of samples using different NGS instruments, sequencing chemistry, gene panels, exome captures and variant calling tools. Fortuitously, during this process we have generated orthogonal validation data using Sanger sequencing for a core set of 142 samples that were included in the majority of our experiments. We now formally use these samples, which we call the ICR142 NGS validation series, to evaluate NGS variant calling performance after any change to experimental or analytical protocols. This series has proved an extremely useful resource for our assessment of NGS analysis in both the research and clinical settings. We believe that it may also have utility for others, and hence are making it available here.

\section{Materials and methods}

We used lymphocyte DNA from 142 unrelated individuals. All individuals were recruited to the BOCS study and have given informed consent for their DNA to be used for genetic research. The study is approved by the London Multicentre Research Ethics Committee (MREC/01/2/18)

Over the last five years we have generated data from the ICR142 validation series using different exome captures which we have analysed with multiple aligner/caller combinations ${ }^{1-6}$. To date we have generated Sanger sequence data for 730 sites amongst the 142 individuals. These sites include variants called by only one aligner and caller combination, increasing the representation of sites which can discriminate performance between methods.

To generate the Sanger sequence data, we performed PCR reactions using the Qiagen Multiplex PCR kit, and bidirectional sequencing of resulting amplicons using the BigDye terminator cycle sequencing kit and an ABI3730 automated sequencer (ABI PerkinElmer). All sequencing traces were analysed with both automated software (Mutation Surveyor version 3.10, SoftGenetics) and visual inspection.

We considered a site negative for a base substitution if the specific base substitution was not present, resulting in 46 negative base substitution sites. We considered a site negative for an indel if no indel, of any kind, was detected in the sequencing trace, resulting in 275 negative indel sites. We annotated confirmed variants with the HGVS-compliant CSN standard using CAVA (version 1.1.0) according to the transcripts designated in Supplementary table $1^{7}$. There were 123 confirmed base substitution variants and 286 confirmed indel variants (Figure 1, Supplementary table 1).

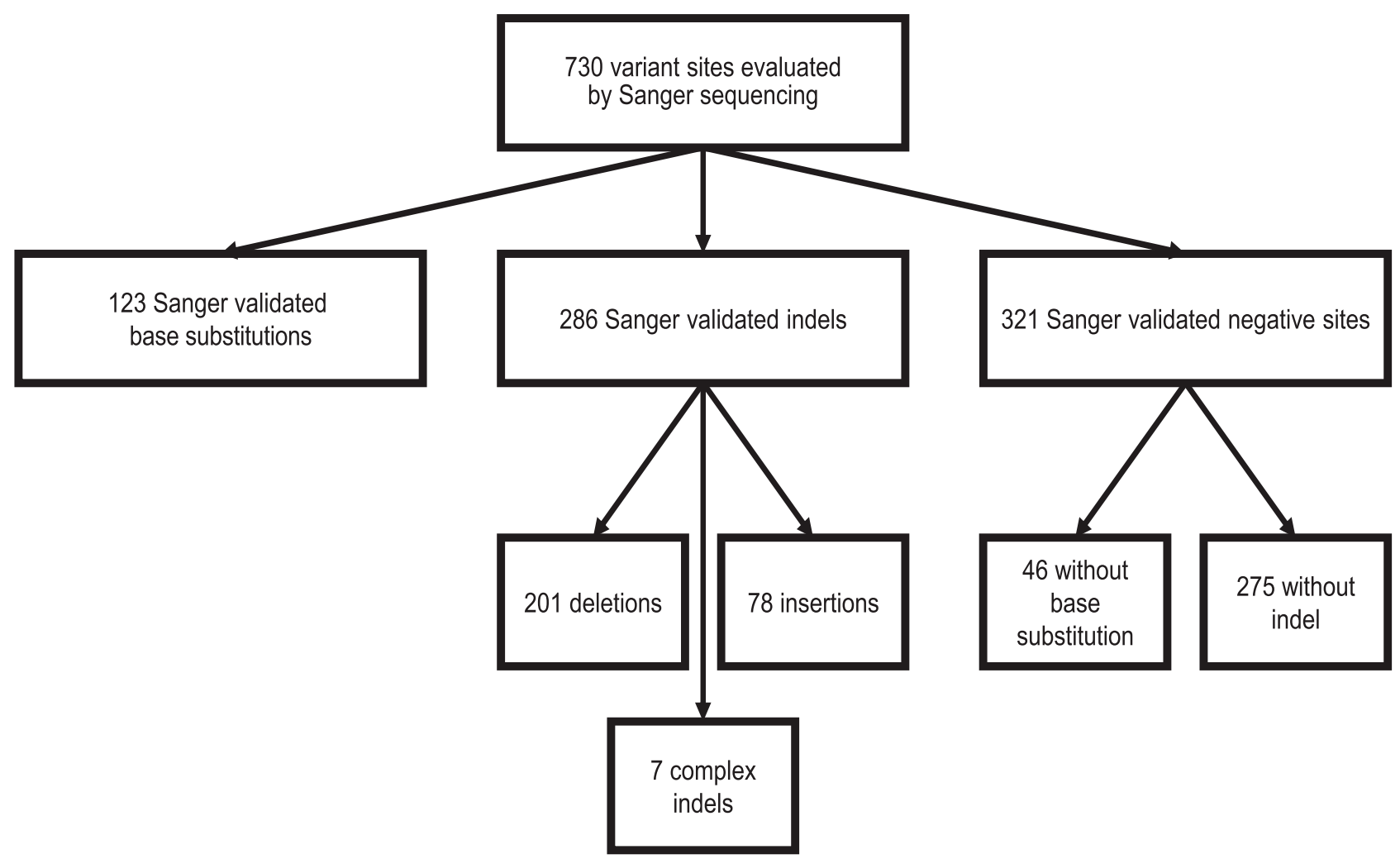

Figure 1. Description of variant sites evaluated by Sanger sequencing in the ICR142 NGS validation series. 
We have also generated high-quality exome sequencing data for the ICR142 NGS validation series. We prepared DNA libraries from $1.5 \mu \mathrm{g}$ genomic DNA using the Illumina TruSeq sample preparation kit. DNA was fragmented using Covaris technology and the libraries were prepared without gel size selection. We performed target enrichment in pools of six libraries (500 ng each) using the Illumina TruSeq Exome Enrichment kit. The captured DNA libraries were PCR amplified using the supplied paired-end PCR primers. Sequencing was performed with an Illumina HiSeq2000 (SBS Kit v3, one pool per lane) generating $2 \times 101$ bp reads. CASAVA v1.8.1 (Illumina) was used to demultiplex and create FASTQ files per sample from the raw base call files.

All of the 730 sites had at least $15 \times$ coverage in the exome data, defined as at least 15 reads of good mapping quality (mapping score $\geq 20$ ). Because these sites are well covered, we can readily assess the variant calling performance of any software tool by applying the pipeline to the exome sequencing data and comparing the variant calls with the Sanger sequencing dataset.

\section{Data availability}

We have deposited the FASTQ files for all 142 individuals in the European Genome-phenome archive (EGA). The accession number is EGAS00001001332. Details of how to request access to the data are available at: www.icr.ac.uk/icr142.

Researchers and authors that use the ICR142 NGS validation series should reference this paper and should include the following acknowledgement: "This study makes use of the ICR142 NGS validation series data generated by Professor Nazneen Rahman's team at The Institute of Cancer Research, London”.

\section{Author contributions}

N.R. and E.Ru. designed the experiment. A.R., E.Ra. and SH generated the exome data. E.Ru. and A.E. undertook data management, S.S., A.R., and K.S. undertook sample management and Sanger validations. M.C. and A.S. undertook the data and administrative management required for data to be accessible. E.Ru. and N.R. wrote the manuscript. All authors contributed to the final manuscript.

\section{Competing interests}

No competing interests were disclosed.

\section{Grant information}

We acknowledge NHS funding to the NIHR Biomedical Research Centre at The Royal Marsden and the ICR. This study was funded by the Institute of Cancer Research, London.

I confirm that the funders had no role in study design, data collection and analysis, decision to publish, or preparation of the manuscript.

\section{Acknowledgements}

We are grateful to the Scientific Computing Team at the Institute of Cancer Research for provision of HPC services. We are grateful to Peter Humburg, Andy Rimmer, Manuel Rivas and Peter Donnelly for undertaking some of the aligner/caller comparisons.

\section{Supplementary material}

Supplementary table 1. Sanger sequencing results for 730 sites in the ICR142 NGS validation series. Confirmed variants are annotated according to the designated transcript by CAVA using $\mathrm{CSN}^{7}$.

The description of the column headings are given below:

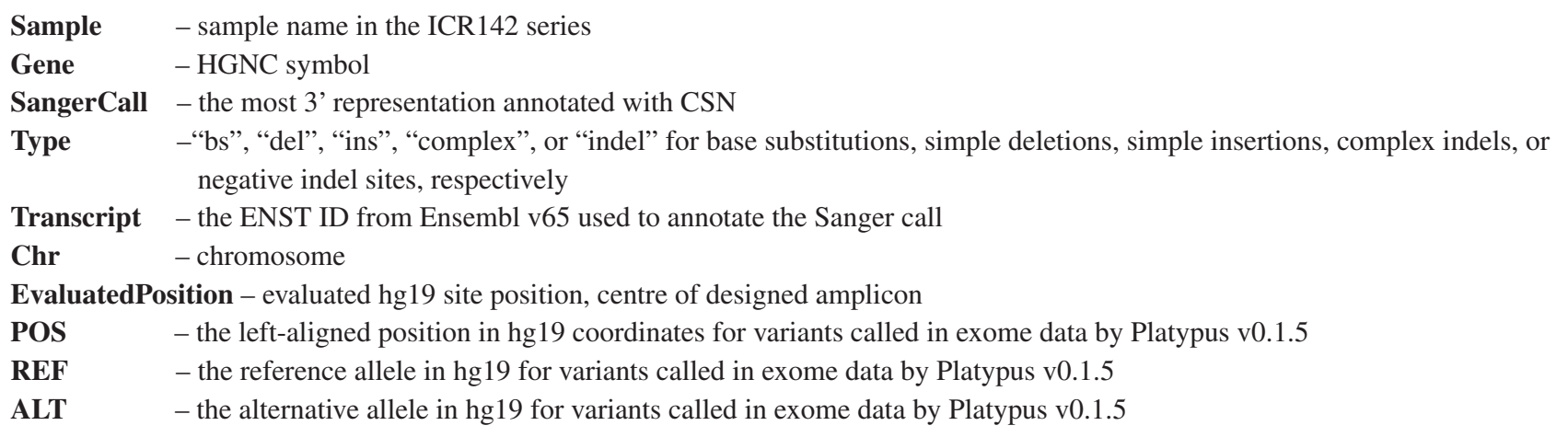


1. Lunter G, Goodson M: Stampy: a statistical algorithm for sensitive and fast mapping of Illumina sequence reads. Genome Res. 2011; 21(6): 936-9. PubMed Abstract | Publisher Full Text | Free Full Text

2. Li H, Durbin R: Fast and accurate short read alignment with Burrows-Wheeler transform. Bioinformatics. 2009; 25(14): 1754-60. PubMed Abstract | Publisher Full Text | Free Full Text

3. Rivas MA, Beaudoin M, Gardet A, et al:: Deep resequencing of GWAS loci identifies independent rare variants associated with inflammatory bowel disease. Nat Genet. 2011; 43(11): 1066-73.

PubMed Abstract | Publisher Full Text | Free Full Text

4. McKenna A, Hanna M, Banks E, et al: The Genome Analysis Toolkit: a MapReduce framework for analyzing next-generation DNA sequencing data.
Genome Res. 2010; 20(9): 1297-303.

PubMed Abstract | Publisher Full Text | Free Full Text

5. Rimmer A, Mathieson I, Lunter G, et al.: Platypus: An Integrated Variant Caller. 2012.

Reference Source

6. SOFTGENETICS: NextgGENe® software for Next Generation (NGS) sequence analysis.

Reference Source

7. Münz M, Ruark E, Renwick A, et al:: CSN and CAVA: variant annotation tools for rapid, robust next-generation sequencing analysis in the clinical setting. Genome Med. 2015; 7(1): 76.

PubMed Abstract | Publisher Full Text | Free Full Text 


\section{Open Peer Review}

\section{Current Peer Review Status:}

\section{Version 1}

Reviewer Report 03 May 2016

https://doi.org/10.5256/f1000research.8841.r13013

(C) 2016 Chapman B et al. This is an open access peer review report distributed under the terms of the Creative Commons Attribution License, which permits unrestricted use, distribution, and reproduction in any medium, provided the original work is properly cited.

\section{Brad Chapman}

Department of Biostatistics, Harvard Public School of Health, Boston, MA, USA

\section{Oliver Hofmann}

Wolfson Wohl Cancer Research Centre, Institute of Cancer Sciences, University of Glasgow, Glasgow, UK

The authors describe ICR142, a publicly available set of fastq files and confirmed true and false variants for validating analysis pipelines. This is an incredibly useful community resource that complements existing efforts like the Genome in a Bottle project by providing a set of validated, difficult regions to evaluate variant detection tools. I appreciate the efforts to make these test sets public; instead of having validation sets like these developed internally at clinical laboratories, we can collaborate and improve them publicly.

In collaboration with Oliver Hofmann at the Wolfson Wohl Cancer Research Center ( https://twitter.com/fiamh) we obtained access to the data and were able to run a validation using bcbio variant calling (http://bcbio-nextgen.readthedocs.io). In doing this, we tried to address a couple of challenges for other users wanting to make immediate use of this data in their own in hour validation work:

The truth sets are not easy to plug into existing validation frameworks. Most validation tools like rtg vcfeval and hap.py work from VCF format files, while this truth set is in a custom spreadsheet format with a mixture of methods for describing changes. You can use Platypus positions for many but need to use CSN descriptions or evaluated position for the remainder.

The truth sets don't appear to describe if we expect calls to be homozygous or heterozygous calls at each position.

Many existing validation approaches expect a single (or few) samples so coordinating checking and validation for all these samples can be a challenge.

As part of this review, we generated a set of configuration files and scripts to help make 
running validations with ICR142 easier (https://github.com/bcbio/icr142-validation). This comparison work also includes a set of comparisons with common callers (GATK HaplotypeCaller, FreeBayes and VarDict). Several of the Sanger validated regions without variants are false positives in at least 2 of the callers tested, so this dataset exposes some common issues with calling and filtering. It would be useful to hear the author's experience with validating callers using this benchmark set and if they have additional filters used to avoid these problems. Knowing a baseline expectation for results would help ensure that the users understand how correctly they've setup the validation resources.

Competing Interests: No competing interests were disclosed.

We confirm that we have read this submission and believe that we have an appropriate level of expertise to confirm that it is of an acceptable scientific standard.

Reviewer Report 21 April 2016

https://doi.org/10.5256/f1000research.8841.r13347

(C) 2016 Bagnall R. This is an open access peer review report distributed under the terms of the Creative Commons Attribution License, which permits unrestricted use, distribution, and reproduction in any medium, provided the original work is properly cited.

\section{Richard Bagnall}

Agnes Ginges Centre for Molecular Cardiology, Centenary Institute, Sydney Medical School, The University of Sydney, Sydney, NSW, Australia

A myriad of software tools have been developed for the alignment of next generation sequencing data to a reference genome and for the subsequent genotyping of DNA variants. Evaluating the specificity and sensitivity of a variant calling framework can be achieved with a dataset containing validated genotypes. Ruark et. al. provide the 'ICR142 NGS validation series' exome sequence fastq files of 142 individuals, and a large set of corresponding Sanger sequencing validated variant sites and sites where variants were called by an NGS tool, but no variant was found with the corresponding Sanger sequencing.

I found the NGS dataset to be easily accessible, on request, from the European Genome-phenome archive and it comprises paired end fastq sequencing files generated by an Illumina sequencing system on the stated 142 individuals. The Sanger sequencing dataset is available as supplementary table 1 of the manuscript. This is a useful resource for evaluating variant calling pipelines.

Competing Interests: No competing interests were disclosed.

I confirm that I have read this submission and believe that I have an appropriate level of expertise to confirm that it is of an acceptable scientific standard. 
The benefits of publishing with F1000Research:

- Your article is published within days, with no editorial bias

- You can publish traditional articles, null/negative results, case reports, data notes and more

- The peer review process is transparent and collaborative

- Your article is indexed in PubMed after passing peer review

- Dedicated customer support at every stage

For pre-submission enquiries, contact research@f1000.com 